Pol. Con. (Edición núm. 18) Vol. 3, No 4

Abril 2018, pp. 185-196

ISSN: 2550 - 682X

DOI: 10.23857/pc.v3i4.480

Ciencias de Educación

Artículo de Revisión

Recepción: 12/ 01/ 2018

Aceptación: 25 / 02/ 2018

Publicación: 21/ 04/2018

\title{
Los derechos humanos en la formación universitaria ecuatoriana
}

\section{Human rights in the Ecuadorian university education}

\section{Direitos humanos na educação universitária equatoriana}

\author{
Leo Ruperti-León \\ leoruperti@hotmail.com \\ Julia R. Morales-Loor \\ julyamores@hotmail.com \\ Néstor D. Suárez-Montes \\ director@dominiodelasciencias.com
}

\section{Correspondencia: leoruperti@hotmail.com}

\footnotetext{
${ }^{\text {I }}$ Magister en Gerencia Educativa, Licenciado en Ciencias Sociales y Políticas, Abogado de los Juzgados y Tribunales de La República, Docente en la Universidad Laica "Eloy Alfaro" de Manabí, Manta, Ecuador.

II Magister en Derecho Constitucional, Diploma Superior en Derecho Procesal Penal, Licenciado en Ciencias Políticas y Sociales, Abogado de los Juzgados y Tribunales de la República. Docente de la Universidad San Gregorio de Portoviejo, Portoviejo, Ecuador.

III Licenciado en Ciencias Sociales y Políticas, Abogado de los Juzgados y Tribunales de la República, Polo de Capacitación, Investigación y Publicación, POCAIP, Manta, Ecuador.
} 


\title{
Resumen
}

Desde siempre los hombres y las mujeres han luchado por sus derechos, como satisfacción de necesidades para alcanzar el pleno desarrollo de la vida y la dignidad humana. Sin embargo, es notorio observar entre los profesionales universitarios, poco interés en el conocimiento pleno de los principios y argumentos en materia de derechos humanos, hecho que se materializa en el ejercicio de su profesión, mermando la posibilidad de convertirse en profesionales proactivos. En este sentido, el estudio evaluó el uso y la formación en derechos humanos de los universitarios ecuatorianos. Se utilizó una metodología descriptiva de campo. La obtención de la información, se obtuvo posterior a la aplicación de un instrumento tipo cuestionario a estudiantes universitarios egresados. Posterior al análisis de la información y datos, se pudo concluir que existe acuerdo en opinión de los investigados, en que privan en su acción profesional el uso de los principios doctrinario del valor Libertad y Justicia Social y un ni acuerdo ni en desacuerdo con la consideración de los principios doctrinarios del valor de Igualdad y Dignidad durante el ejercicio de su profesión.

Palabras clave: derechos humanos; principios doctrinarios; profesionales universitarios.

\begin{abstract}
Men and women have always fought for their rights, as satisfaction of needs to achieve the full development of life and human dignity. However, it is notorious to observe among university professionals, little interest in full knowledge of the principles and arguments in the field of human rights, a fact that materializes in the exercise of their profession, diminishing the possibility of becoming proactive professionals. In this sense, the study evaluated the use and training in human rights of Ecuadorian university students. A descriptive field methodology was used. The obtaining of the information was obtained after the application of a questionnaire-type instrument to graduated university students. After the analysis of the information and data, it was concluded that there is agreement in the opinion of the investigated, in which they deprive in their professional action the use of the doctrinal principles of the value of Freedom and Social Justice and a neither agreement nor in disagreement with the consideration of the doctrinal principles of the value of Equality and Dignity during the exercise of their profession.
\end{abstract}

Keywords: human rights; doctrinal principles; university professionals. 


\section{Resumo}

Homens e mulheres sempre lutaram por seus direitos, como satisfação de necessidades para alcançar o pleno desenvolvimento da vida e da dignidade humana. No entanto, é notável observar entre os profissionais universitários, pouco interesse no pleno conhecimento dos princípios e argumentos sobre direitos humanos, um fato que está incorporada no exercício de sua profissão, minando a possibilidade de se tornar profissionais pró-ativos. Nesse sentido, o estudo avaliou o uso e treinamento em direitos humanos de universitários equatorianos. Uma metodologia de campo descritiva foi utilizada. A obtenção das informações foi obtida após a aplicação de um instrumento do tipo questionário para estudantes universitários graduados. Após a análise de informações e dados, concluiu-se que há um acordo tendo em vista a investigação, que prevalecem em sua ação profissional, utilizando os princípios doutrinários da liberdade e valor da justiça social e não concordo nem discordo com a consideração dos princípios doutrinais do valor da igualdade e da dignidade durante o exercício da profissão.

Palavras chave: direitos humanos; princípios doutrinais; profissionais universitários. 


\section{INTRODUCCIÓN}

Universalmente, los derechos humanos constituyen un conjunto de principios, de aceptación universal orientados a asegurar al ser humano su dignidad como persona, en su dimensión individual, social, material y espiritual, los cuales el Estado está en el deber de respetar, garantizar o satisfacer. Asimismo, la Declaración Universal de Derechos Humanos la define como el conjunto de reglas encaminadas a proteger el conjunto de los derechos civiles y políticos, así como los derechos económicos, sociales, ambientales y culturales de las personas.

Baratta (2004), explica que se debe hablar de derecho humanos a partir de un concepto integrado por los elementos: hombre y derecho, en la que ambos están integrados a partir de una relación de complementariedad y de contradicciones. El primero, en tanto que el derecho le pertenece al hombre como es y de contradicciones cuando el derecho no reconoce al hombre lo que le pertenece, lo que hace complejo tener una definición de derechos humanos. Asimismo, Pérez Luño (2007), la considera como un conjunto de facultades institucionales que, en cada momento histórico, concreta exigencias de la dignidad, la libertad, y la igualdad humana, las cuales deben ser reconocidas positivamente por los ordenamientos jurídicos a nivel nacional o internacional., siendo así, estas facultades que conforme al derecho internacional, tiene todo individuo frente a los órganos del poder, han de permitir preservar su dignidad como ser humano y excluir la interferencia del Estado en áreas específicas de la vida individual, y/o asegurar la prestación de determinados servicios por parte del Estado, para satisfacer sus necesidades básicas.

Cabe señalar, que el propósito general para el sistema educativo ecuatoriano es contar con una malla curricular de educación inspirada en principios éticos, pluralistas, democráticos, humanistas y científicos, que promueva el respeto a los derechos humanos, derechos colectivos, desarrolle un pensamiento crítico, fomente el civismo; proporcione destrezas para la eficiencia en el trabajo y la producción; estimule la creatividad y el pleno desarrollo de la personalidad y las especiales habilidades de cada persona; impulse la interculturalidad, la solidaridad y la paz. Una educación que prepare a ciudadanos para la producción de conocimientos y para el trabajo.

Por otro lado, la Ley de Educación Superior vigente, contiene aspectos muy avanzados con respecto a la formación de los estudiantes universitarios y el respeto a sus derechos y los derechos humanos, con los instrumentos internacionales de derechos humanos que regulan los principios sobre educación superior; con los nuevos desafíos del Estado ecuatoriano que busca 
formar profesionales y académicos con una visión humanista, solidaria, comprometida con los objetivos nacionales y con el buen vivir, en un marco de pluralidad y respeto. Sin embargo, es notorio en los profesionales universitarios un desconocimiento en materia de derechos humanos, visibilizado en su accionar profesional, específicamente en el desconocimiento de la otredad. Al respeto, en este trabajo se identificaron los principios doctrinarios que distinguen los derechos humanos en la acción profesional de los egresados universitarios ecuatorianos.

\section{DESARROLLO}

\section{Principios doctrinarios en los derechos humanos.}

El Estado ha de sustentarse, según Araujo (1999), en los valores de igualdad, dignidad, libertad, y justicia social, como garantía del cumplimiento del gran principio del respeto de la distribución y redistribución de los productos y bienes sociales, para vivir bien.

\section{Principio doctrinario del valor Libertad.}

El valor libertad, puede ser estudiado desde dos perspectivas; una de tipo organizativa y otra relacionada con el status de las personas en la organización social. En la primera, se entiende que la libertad es la base fundamental de la organización del Estado y organización social, que se expresa a través del texto constitucional; ..."implica entonces la soberanía popular, legitimación de los gobernantes por medio de elecciones periódicas por sufragio universal; sujeción de los poderes públicos y de los ciudadanos a la ley, y reconocimiento y protección de los derechos fundamentales (Rivera, 2009:359)". En la segunda relacionada con el status de las personas en la organización estatal y social, la libertad implica básicamente la libertad de autonomía, la libertad de participación y la libertad de prestación. De este modo, la inclusión de la libertad como valor supremo, exige que exista una protección garantizada y reconocida por el Estado de la libertad de cada uno, dentro de los límites que señalan la Constitución y las leyes.

De lo afirmado se deduce, que la libertad debe entenderse como la capacidad jurídica del individuo de hacer todo lo que no perjudicase a otro. Sólo esta exigencia de garantizar la libertad ajena, puede constituir el límite de la libertad individual y esto debe ser fijado por la ley. Por lo tanto, la ley no tiene el derecho de prohibir más que las acciones perjudiciales a la sociedad; por lo demás, todo lo que no está prohibido por la ley está permitido y, nadie puede ser obligado a hacer lo que ella no ordene. En esta perspectiva, lo que se busca es que el individuo goce de una 
esfera de derechos impenetrables, los cuales le permitan participar como elector o elegible en la formación del poder público y estos se encuentren debidamente garantizados a objeto de obtener las prestaciones necesarias por parte del Estado.

\section{Principio doctrinario del valor Igualdad.}

El valor igualdad, ha de responder a la idea de que. "no basta con proteger la libertad individual, sino que hay que lograr el objetivo más ambicioso: asegurar una igual eficacia formal y material de la ley para todos, proscribir cualquier tipo de discriminación entre personas y grupos sin negar la previsible diferenciación basada en motivos objetivos, y, procurar que exista una seguridad mínima para todos los ciudadanos respecto a las condiciones de vida material (Lopez, 1980:52)". De ello se infiere, que el papel del Estado no debe reducirse a resguardar el orden público y no interferir ni vulnerar las libertades de las personas, sino principalmente a contrarrestar las diferencias sociales existentes y ofrecer a todos sus habitantes las oportunidades necesarias para desarrollar sus aptitudes y para superar las necesidades materiales.

\section{Principio doctrinario del valor Dignidad.}

Con relación al valor dignidad, se puede entender que es aquél que tiene toda persona por su sola condición de humano, para que se le respete y reconozca como un ser dotado de un fin propio, y no como un medio para la consecuencia de fines extraños o autoritarios, ajenos a su realización personal. En ese entendido, el valor supremo de dignidad, inexorablemente debe ser entendido como: el derecho de toda persona a un trato que no lesione su condición de ser racional, libre igual y capaz de autodeterminación responsable; lo que conlleva la prohibición de que sea tratado como un objeto o instrumento. De ello, surge en primer término, un mandato de abstención a los poderes públicos y en lo pertinente a los particulares, que prohíbe la producción de normas o la realización de actos, que tengan un contenido degradante o envilecedor; y en segundo término, un mandato de actuación, que le incline a desarrollar políticas destinadas a promocionar o favorecer el desarrollo de la persona. Por tanto, se lesionará la dignidad humana, todo acto o disposición que degrade o envilezca a la persona a un nivel de estima incompatible con su naturaleza humana, cualquiera sea el lugar o la situación en la que se encuentre. Por esta 
razón, la dignidad humana conjuntamente los derechos humanos, encierran conceptos universales capaces de traspasar cualquier sistema político social.

La dignidad se basa en el reconocimiento de la persona de ser merecedora de respeto, es decir que todos merecemos respeto sin importar cómo seamos. Al reconocer y tolerar las diferencias de cada persona, para que ésta se sienta digna y libre, se afirma la virtud y la propia dignidad del individuo, fundamentado en el respeto a cualquier otro ser. En este sentido, Thomas (2007), la define como la excelencia que merece respeto o estima, tal es el caso de una persona que ocupe un alto rango o un puesto elevado y posee una dignidad, lo que exige a los demás una respuesta particular, pero esto no le hace acreedor a una mayor dignidad que el resto, ya que ésta, es igual para todos los seres humanos, sin importar su condición o puesto que desempeñe. De ahí que deba existir una relación entre dignidad humana y los derechos el hombre.

\section{Principio doctrinario del valor Justicia social.}

Con relación al valor justicia social, se puede entender bajo la máxima que consiste en dar a cada uno lo suyo; este valor se encuentra ligado a la dignidad de la persona. La justicia debe abarcar la totalidad del orden jurídico, es decir, que todo el ordenamiento jurídico por regla imperativa deberá intentar ser lo más justo posible, dado que conforme exista la materialización de la justicia mediante el derecho positivizado, se logrará garantizar un orden político, económico y social justo y equitativo. En tanto el derecho más se apegue al valor justicia, el Estado Constitucional se encontrará distante del autoritarismo.

En materia constitucional, los valores cumplen un rol preponderante en el sentido que le imprimen a la Constitución y sobre todo al orden jurídico estatal y comunitario, de tal modo que estos se encuentran ligados a una concepción democrática y uno de sus objetos en un sistema democrático es: aspirar a un determinado régimen constitucional, en donde caben ciertamente numerosas tendencias e ideologías de carácter opuesto, pero en donde no es posible una evolución basada incluso en la legalidad, hacia un régimen en que no se respetasen tales valores. Así, no sería legítimo entender incluso otras normas incluidas en la propia Constitución en un sentido opuesto a ellos. Sin embargo, para Hortal (2004), este principio está estrechamente ligado con el sentido social de la profesión. En tanto que, es el colectivo profesional, quienes se hacen 
responsables ante la sociedad de los bienes y servicios que buscan promover y que se traducen en un compromiso a favor del bien público.

\section{Análisis de los resultados}

Posterior al análisis de los datos aportado por los profesionales investigados, los mismos se presentan en tablas con su respectiva interpretación.

Tabla $\mathbf{N}^{\mathrm{a}} 1$

Distribución frecuencial y porcentual del indicador principio doctrinario del valor Justicia Social.

\begin{tabular}{ccccc}
\hline ANÁLISIS/ CRITERIOS & FR & $\mathbf{\%}$ & $\boldsymbol{F R A}$ & $\mathbf{\% A}$ \\
\hline Totalmente de acuerdo & 5 & 18,5 & 5 & 18,5 \\
\hline De acuerdo & 12 & 44,4 & 17 & 62,9 \\
\hline Ni de acuerdo ni en desacuerdo & 8 & 29,7 & 25 & 92,6 \\
\hline En desacuerdo & 2 & 7,4 & 27 & 100 \\
\hline Totalmente en desacuerdo & 0 & 0 & 27 & 100
\end{tabular}

\section{Fuente: Calculo del Autor.}

En la tabla 1, se presentan los resultados del análisis frecuencial del indicador principio doctrinario del valor Justicia Social, en la misma se señala que el 44,4\% del total de los investigados, coincidieron en opinar que se encontraban de acuerdo con que asumen una conducta profesional en términos de la imparcialidad y del ejercicio de la equidad, para el mantenimiento de lo que es correcto, así como en proporcionar relaciones justas de trabajo entre el personal adscrito a su dependencia. Seguido del 29,7\% y 18,5\% que señalaron las alternativas ni de acuerdo ni en desacuerdo y totalmente en desacuerdo respectivamente. El menor porcentaje se indicó para la alternativa en desacuerdo con el 7,4\% del total de las opiniones. Se destaca el hecho, en la cual la alternativa totalmente en desacuerdo, que más desfavorece la evaluación del indicador no presento opinión alguna.

De acuerdo a los resultados obtenidos, se puede observar que los mismos se acercan a lo propuesto por Hortal (2004), en cuanto a que este principio está estrechamente ligado con el 
sentido social de la profesión. En tanto que, es el colectivo profesional, quienes se hacen responsables ante la sociedad de los bienes y servicios que buscan promover y que se traducen en un compromiso a favor del bien público.

Tabla No. 2

Distribución frecuencial y porcentual del indicador principio doctrinario del valor Libertad.

\begin{tabular}{ccccc}
\hline ANÁLISIS/ CRITERIOS & FR & \% & FRA & \%A \\
\hline Totalmente de acuerdo & 5 & 18,5 & 5 & 18,5 \\
\hline De acuerdo & 14 & 51,9 & 19 & 70,4 \\
\hline Ni de acuerdo ni en desacuerdo & 8 & 29,6 & 27 & 100 \\
\hline En desacuerdo & 0 & 0 & 27 & 100 \\
\hline Totalmente en desacuerdo & 0 & 0 & 27 & 100
\end{tabular}

\section{Fuente: Calculo del Autor.}

En la tabla 2, se presentan los resultados del análisis frecuencial del indicador principio doctrinario del valor libertad, en la misma se señala que el 51,9\% del total de los investigados, coincidieron en opinar que se encontraban de acuerdo en que actúan fundamentados en la libertad de autonomía, de participación y de prestación. De este modo, la inclusión de la libertad como valor supremo, también exigen que exista una protección garantizada y reconocida de su libertad dentro de los límites que señalan la Constitución y las leyes. Seguido del 29,6\% que señalaron la alternativa ni de acuerdo ni en desacuerdo. El menor porcentaje se indicó para la alternativa totalmente de acuerdo con el $18,5 \%$ del total de las opiniones. Se destaca el hecho, en la cual las alternativas en desacuerdo y totalmente en desacuerdo, que más desfavorece la evaluación del indicador, no presentaron opinión alguna. En este sentido, cabe destacar que la libertad debe entenderse como la capacidad jurídica del individuo de hacer todo lo que no perjudicase a otro. Sólo esta exigencia de garantizar la libertad ajena, puede constituir el límite de la libertad individual y esto debe ser fijado por la ley. 
Tabla No. 3

Distribución frecuencial y porcentual del indicador principio Doctrinario del valor Igualdad.

\begin{tabular}{ccccc}
\hline ANÁLISIS/ CRITERIOS & FR & \% & FRA & \%A \\
\hline Totalmente de acuerdo & 0 & 0 & 0 & 0 \\
\hline De acuerdo & 5 & 18,5 & 5 & 18,5 \\
\hline Ni de acuerdo ni en desacuerdo & 15 & 55,6 & 20 & 74,1 \\
\hline En desacuerdo & 7 & 25,9 & 27 & 100 \\
\hline Totalmente en desacuerdo & 0 & 0 & 27 & 100
\end{tabular}

Fuente: Calculo del Autor.

En la tabla 3, se presentan los resultados del análisis frecuencial del indicador principio doctrinario del valor igualdad, en la misma se señala que el 55,6\% del total de los investigados, coincidieron en opinar que se encontraban ni de acuerdo ni en desacuerdo en que durante el ejercicio de sus profesión se garantizara la igualdad de aplicación de la ley para todos, en ocasiones se proscribían algún tipo de discriminación entre personas y grupos profesionales. Seguido del 18,5\% y 25,9\% que señalaron las alternativas de acuerdo y en desacuerdo respectivamente. Se destaca el hecho, en la cual las alternativas extremas de totalmente de acuerdo y totalmente en desacuerdo no presentaron opinión alguna entre los encuestados.

Al respecto, López (1980), indica que el papel del Estado no debe reducirse a resguardar el orden público y no interferir ni vulnerar las libertades de las personas, sino principalmente a contrarrestar las diferencias sociales existentes y ofrecer a todos sus habitantes las oportunidades necesarias para desarrollar sus aptitudes y para superar las necesidades materiales. 
Tabla No. 4

Distribución frecuencial y porcentual del indicador principio

Doctrinario del valor Dignidad.

\begin{tabular}{ccccc}
\hline ANÁLISIS/ CRITERIOS & FR & \% & FRA & \%A \\
\hline Totalmente de acuerdo & 0 & 0 & 0 & 0 \\
\hline De acuerdo & 6 & 22,2 & 6 & 22,2 \\
\hline Ni de acuerdo ni en desacuerdo & 13 & 48,2 & 19 & 70,4 \\
\hline Totalmente en desacuerdo & 0 & 0 & 27 & 100
\end{tabular}

\section{Fuente: Calculo del Autor.}

En la tabla 4, se presentan los resultados del análisis frecuencial del indicador principio de Doctrinario del valor Dignidad, en la misma se señala que el 48,2\% del total de los investigados, coincidieron en opinar que se encontraban ni de acuerdo ni en desacuerdo que durante el ejercicio de su profesión han sido lesionados su condición de ser racional, libre, igual y capacidad de autodeterminación responsable, sintiéndose tratado como un objeto o instrumento. Seguido del $22,2 \%$ y $29,6 \%$ que señalaron las alternativas de acuerdo y en desacuerdo respectivamente. Se destaca el hecho, en la cual las alternativas extremas de totalmente de acuerdo y totalmente en desacuerdo no presentaron opinión alguna entre los encuestados.

De acuerdo a los resultados obtenidos se tiene que los mismos se alejan del reconocimiento que toda persona merece a través del respeto, es decir que todos han de merecer respeto sin importar cómo seamos. Al reconocer y tolerar las diferencias de cada persona, para que ésta se sienta digna y libre, se afirma la virtud y la propia dignidad del individuo, fundamentado en el respeto a cualquier otro ser.

\section{Conclusiones}

Se constató un acuerdo entre las opiniones de los profesionales investigados, en que asumen una conducta profesional en términos de la imparcialidad y del ejercicio de la equidad, para el mantenimiento de lo que es correcto, así como en proporcionar relaciones justas de trabajo entre el personal adscrito a su dependencia y actúan fundamentados en la libertad de autonomía, de participación y de prestación. 
Se evidencio, un ni de acuerdo ni en desacuerdo, que durante el ejercicio de su profesión se les garantiza la igualdad de aplicación de la ley para todos y en que se les lesiona su condición de ser racional, libre, igual y con capacidad de autodeterminación responsable, en la que se podrían sentir tratado como un objeto o instrumento.

\section{Referencias Bibliográficas}

ARAUJO, JAIME (1999). Principios del Derecho Constitucional. Bogotá, Ed. Mc Graw Hill.

BARATTA, ALESSANDRO, (2004). Criminología y Sistema Penal (Compilación in memoriam), Editorial B de F, Buenos Aires, Argentina.

HORTAL, AUGUSTO (2004). Ética general de las profesiones. Segunda Edición. BilbaoEspaña. Editorial Desclée de Brouwer.

LOES (2010). Ley Orgánica de Educación Superior del Ecuador.

LOPEZ, LUÍS; DE ESTEBAN, JORGE (1980). El régimen constitucional español. Barcelona, Ed. Labor universitaria.

PEREZ LUDINO (2007). Los Derechos Fundamentales. Madrid. Tecnos. ISBN 978-84-309$\underline{4557-3}$

RIVERA SANTIVAÑEZ, JOSÉ ANTONIO (2009). Los valores supremos y principios fundamentales en la jurisprudencia del Tribunal Constitucional. Ob. cit. p. 359.

THOMAS WILLIAMS,(2007) Fundamentos de los Derechos del Hombre y el Principio Rector del Bien Común, en la página web http://www.catolicos.com/socialdoc12.htm . 